\title{
Meynert's Nucleus Complex White Matter Abnormalities in Autism Spectrum Disorders: An MRI Study
}

\author{
Matteo Pardini ${ }^{1,2}$, Francesco G. Garaci ${ }^{3}$, Laszlo Zaborszky ${ }^{4}$, Filadelfo Coniglione ${ }^{5}$, \\ Gianluca Serafini ${ }^{1}$, Martina Siracusano ${ }^{6}$, Francesca Benassi ${ }^{2}$ and \\ Leonardo Emberti Gialloreti ${ }^{2,3, *}$
}

${ }^{1}$ Department of Neurosciences, Rehabilitation, Ophthalmology, Genetics and Maternal and Child Health, University of Genoa, Largo Daneo 3, IT-16132 Genoa, Italy

${ }^{2}$ Centre for Communication and Neurorehabilitation Research-CNAPP, Via Marcantonio Boldetti 12, 00162 , Rome, Italy

${ }^{3}$ Department of Biomedicine and Prevention, University of Rome Tor Vergata, Via Montpellier 1, 00133 , Rome, Italy

${ }^{4}$ Centre for Molecular and Behavioural Neuroscience, Rutgers, The State University of New Jersey, 57 US Highway 1 New Brunswick, NJ 08901-8554 USA

${ }^{5}$ Department of Clinical Sciences and Translational Medicine, University of Rome Tor Vergata, Via Montpellier 1, 00133, Rome, Rome, Italy

${ }^{6}$ Department of Neuroscience, Paediatric Neurology Unit, University of Rome Tor Vergata, Via Montpellier 1, 00133, Rome, Italy; Rome, Italy

\begin{abstract}
Introduction: Cholinergic dysfunction has been proposed to play a role in autistic symtomatology. However, to date, its structural correlates are poorly understood.

Methods: Twenty-five low-functioning, non-verbal males with Autism Spectrum Disorders (ASD) and 25 controls were enrolled in the study. All underwent MR T1-weighted 3D Structural Imaging and Diffusion Tensor Imaging. Grey and white matter components of the Meynert's Nucleus Complex were then identified on MR images, and both grey matter density and white matter mean Fractional Anisotropy in the Meynert's Nucleus region of interest were quantified for each subject. Non-verbal IQ was assessed in all subjects with ASD.

Results: We showed reduced white matter Fractional Anisotropy in the bundles surrounding the Meynert's Nucleus in ASD subjects compared to controls. Fractional Anisotropy in these bundles was positively associated with non-verbal IQ, independently from whole brain white matter mean Fractional Anisotropy. ASD subjects did not show significant abnormalities in Meynert's Nucleus grey matter density.

Conclusions: Our findings suggest that white matter abnormalities in the Meynert's Nucleus might be involved in the cholinergic deficits of ASD.
\end{abstract}

Keywords: Autism, cholinergic system, Meynert's nucleus, diffusion tensor imaging, white matter.

\section{INTRODUCTION}

Autism Spectrum Disorders (ASD) are neurodevelopmental syndromes characterized by persistent deficits in social communication and social interaction and by restricted, repetitive patterns of behaviour, interests, or activities, causing clinically significant impairment in social, occupational, or other important areas of functioning [1]. While the biological bases of ASD are only partially understood, a wide range of experimental and clinical observations suggest that cholinergic system alterations could be involved in these disorders [2-7]. That is why some

*Address correspondence to this author at the Department of Biomedicine and Prevention. University of Rome Tor Vergata, via Montpellier 1, 00133, Rome, Italy; Tel: +39.0672596614; Fax: +39.0620427263;

E-mail: leonardo.emberti.gialloreti@uniroma2.it authors have proposed that the nicotinic cholinergic system might be involved in the pathogenesis of ASD [8]. Pathologic studies have indeed highlighted that nicotinic acetylcholine receptor subtypes are altered in brain of autistic individuals. In fact, individuals with ASD often show altered sensory processing, which could be one of the underlying reasons of its core symptoms. These observations led to examine the role of the nicotinic cholinergic system on social and repetitive behavior abnormalities and exploratory physical activity in models of autism [5]. Several of findings support therefore the hypothesis that the nicotinic cholinergic system modulates social and repetitive behaviours and that it might become in future a possible therapeutic target to treat behavior deficits in ASD.

In addition, some researches have suggested that cholinergic activity might also play a role in cognitive 
processes, such as attention, which are often altered in this population [9]. It has been therefore proposed that the attentional deficits observed in ASD might be linked to disruptions of the cholinergic modulation by acting on local cortical microcircuits to disconnect spontaneous activity. Defects in this mechanism might represent endophenotypes that could link diverse genetic and developmental disruptions to attentional deficits in ASD [10].

Notwithstanding these speculations, it has not been fully clarified yet which specific circuits' abnormalities might affect the cholinergic system of subjects with ASD [10]. A loss of cortical and deep grey matter nicotinic acetylcholine receptors has been shown in autistic subjects as well $[4,6]$, leading to preclinical studies, which tested the use of nicotine to reduce aggression-related behaviours [11]. Also treatments with acetylcholinesterase inhibitors have been reported to positively impact on cognitive and behavioural symptomatology in a subset of ASD subjects [2]. In addition, cholinergic abnormalities have been shown in different animal models of autism, and have been associated with repetitive behaviours and social cognition deficits, two of the core features of ASD [5].

The Meynert's Nucleus (nucleus basalis of Meynert) is a broad nucleus in the human forebrain, which presents with projection of cholinergic fibres in nonhuman primates, even if this topography is only partially translatable to the human brain. It is, therefore, important to continue to investigate the role of the Meynert's Nucleus (MN) in the pathophysiology of diseases of the Central Nervous System, ASD included [12]. As a matter of fact, despite this substantial amount of findings, the structural bases of cholinergic dysfunction in people with ASD are still poorly understood.

The first aim of this study was thus to assess the presence of structural abnormalities in the $\mathrm{MN}$, the main human cholinergic nucleus, in low-functioning ASD, using non-conventional MRI techniques. Secondary aim was to shed light on the possible relationship between the structural properties of the $M N$ and non-verbal IQ in ASD.

\section{METHODS}

\section{Subjects' Enrolment and Evaluation}

We recruited 25 low-functioning, right-handed, nonverbal males with ASD, enrolled from the subjects

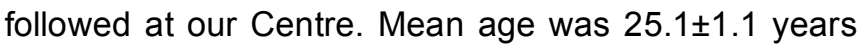

(range: 18-33). All subjects had a diagnosis of ASD according to DSM-5 criteria [1] and none presented with identifiable causes of ASD or neurological comorbidities. Subjects with identifiable causes of autism and known neurological disorders, including epilepsy, were excluded. The 25 included subjects were assessed with the Autism Diagnostic Observation Schedule (ADOS) [13] and the Autism Diagnostic Interview -Revised (ADI-R) [14] before undergoing MRI scanning. ADI-R and ADOS scores, reported in Table 1 , were compatible with a diagnosis of autism for all subjects. Intelligence quotient (IQ) was assessed using the Leiter International Performance Scale-Revised [15], a non-verbal measure of intellectual functioning. Mean nonverbal IQ of the autistic group was $50.9 \pm 0.2$ (range: 39-57). Thirteen subjects had an IQ score below 50 , belonging, therefore, to the severe range of autism. After a complete description of the study to the subjects, parents and/or guardians, written informed consent was obtained. Moreover, 25 healthy males, matched with our ASD group on age (mean age: $24.8 \pm 0.9$ years, range: $18-32$ ), socioeconomic status, and handedness, were enrolled as controls for the analysis of MRI data. None of them had a personal history of neurological disorders or a family history of autism or mental retardation. Our ethics committee approved all the study procedures.

\section{MRI Acquisition}

Subjects with ASD underwent scanning under general anaesthesia. Anaesthesia, always with spontaneous breathing, was induced with intravenous propofol or inhalatory sevoforane. No complications occurred during or after MRI scanning. MRI series were obtained on a 3T scanner (Intera Achieva, Philips Medical Systems, Best, the Netherlands) equipped with $80 \mathrm{mT} / \mathrm{m} / \mathrm{ms}$ gradient coils and an 8-channel sensitivity encoding (SENSE) coil. Diffusion Tensor Imaging (DTI) was performed using single-shot spin-echo echo-planar imaging ( $T R=10,000$ msec; $T E=59$ msec; FLIP angle=90; matrix size $=112 \times 112 ; \mathrm{FOV}=224 \mathrm{~mm}$; slice thickness $=2 \mathrm{~mm}$; gap between slices $=0 ; \quad \mathrm{NSE}=3$; SENSE factor $=2 ; b=800 \mathrm{sec} / \mathrm{mm}^{2}$ ). Diffusion gradients were applied in 33 non-collinear directions; moreover, six images without diffusion gradients were acquired for each subject $\left(b=0 \mathrm{sec} / \mathrm{mm}^{2}\right)$. Axial 3D T1-weighted images were acquired for morphological analysis $(\mathrm{TR}=25 \mathrm{msec} ; \mathrm{TE}=2.2 \mathrm{msec} ; \mathrm{FOV}=224 \mathrm{~mm}$; slice thickness $=2 \mathrm{~mm}$; gap between slices $=0$ ).

\section{Meynert's Nucleus Complex Mask (Ch4)}

The MN complex was identified using a mask based on published post-mortem maps of magnocellular cell 
groups of the basal forebrain, reconstructed and registered to the $\mathrm{MNI}$ brain as previously described [16]. Among the magnocellular cell groups masks described in Zaborszky et al. [16], we used in our analyses the Ch4 cell group mask, which corresponds to the MN complex. In MRI images the precise delineation of these structures is difficult, due to limited spatial resolution and contrast. Nevertheless, as probabilistic maps of compartments of the basal forebrain magnocellular system are now available as an open source reference for correlation with fMRI, PET, and structural MRI data of the living human brain, we were able to adopt them to analyse our data [16].

\section{MRI Analysis}

DTI data were processed with the FDT package included in FSL [17]. Pre-processing steps included correction for eddy current distortions and motion artefacts [18]. Fractional Anisotropy (FA) maps were then created with FDT [19]. FA is one of the measures most commonly derived from diffusion data; it quantifies how strongly directional the local tract structure is. Many imaging studies are beginning to use FA images in voxelwise statistical analyses in order to localise brain changes related to development, degeneration and disease. However, optimal analysis is jeopardized by the use of standard registration algorithms, as, until now, there has not been an adequate solution to the question of how to align FA images from multiple subjects in a way that allows for valid conclusions to be drawn from the subsequent voxelwise analysis. We used here the Tract-Based Spatial Statistics (TBSS), so to improve the sensitivity, objectivity and interpretability of the analysis of multisubject diffusion imaging studies [17].

As mentioned, FA is a quantitative index of white matter microstructure: higher FA values are found in more organized white matter tracts. All subjects' FA data were aligned into a common space using the nonlinear registration tool FNIRT included in FSL [17]. Next, using the FSL-TBSS pipeline [17, 19], a group mean FA image was created and thinned to create a mean FA skeleton, which represents all tracts that are common to the group; the individual FA maps were then projected onto the group-wise skeleton to account for residual misalignment and to create individual FA maps of skeletonized white matter [19]. Afterwards, mean FA values were extracted for each subject from those voxels included both in the skeletonized white matter maps and in the Ch4 mask.
Subsequently, morphological data were analyzed using FSL [20]. First, the 3D structural images were brain-extracted using BET and tissue-segmented using FAST4 [20]. The resulting grey-matter partial volume images were then aligned to the MNI152 standard space, using the affine registration tool FLIRT, followed by nonlinear registration using FNIRT [17]. The resulting images were averaged to create a studyspecific template, to which the native grey matter images were non-linearly co-registered. The registered partial volume images were modulated by dividing by the Jacobian of the warp field. Mean grey matter density (GMD) values were then extracted from those voxels included both in the modulated segmented grey matter maps and in the Ch4 mask.

\section{Statistical Analysis}

Mean FA and mean GMD inside the Ch4 mask were firstly compared between controls and autistic subjects using independent samples t-tests.

Mean FA and mean GMD values inside the Ch4 mask were then correlated with non-verbal IQ using Pearson's correlations and partial correlations. Statistical significance was set at $\mathrm{p}=0.05$ (two-tailed). All results are reported as mean \pm standard error of the mean.

\section{RESULTS}

\section{Ch4 Structural Differences between Controls and Subjects with ASD}

Demographic, clinical and MRI data are reported in Table 1. Mean IQ of ASD subjects was about 51. Our subjects can therefore be classified as low-functioning. The high mean ADOS score (23.4), shows that the participating subjects had a manifest diagnosis of ASD.

Inside the Ch4 mask, subjects with ASD presented with significantly lower white matter mean FA values compared to controls $(0.65 \pm 0.01$ vs. $0.70 \pm 0.01$; $t=-$ 4.98, $\mathrm{p}<0.001$ ), while there was no differences between the two groups in mean GMD $(0.59 \pm 0.01$ vs. $0.60 \pm 0.01, t=0.4, p=0.68)$. The next analyses were thus restricted only to white matter mean FA values.

\section{Ch4 and Non-Verbal IQ}

As shown in Figure 1, in the ASD group there was a significant correlation between non-verbal IQ and Ch4 white matter mean FA values $(r=0.58, p<0.002)$; the correlation between IQ and Ch4 FA values remained 
Table 1: Demographic, Clinical and MRI Data of the Enrolled Subjects with ASD and Controls. Measures are given in mean \pm standard error of the mean

\begin{tabular}{|c|c|c|}
\hline & ASD subjects & Control subjects \\
\hline \hline Age (years) & $25.1 \pm 1.1$ & $24.8 \pm 0.9$ \\
\hline ADOS Module 1 (Communication + Social Interaction) & $23.4 \pm 2.3$ & $\mathrm{~N} / \mathrm{A}$ \\
\hline Non-verbal IQ & $50.9 \pm 0.2$ & $\mathrm{~N} / \mathrm{A}$ \\
\hline ADI-R Communication non verbal & $10.4 \pm 1.3$ & $\mathrm{~N} / \mathrm{A} / \mathrm{A}$ \\
\hline ADI-R Social Interaction & $18.4 \pm 1.0$ & $\mathrm{~N} / \mathrm{A}$ \\
\hline ADI-R- Restricted, Repetitive Behaviours & $5.3 \pm 2.6$ & $0.70 \pm 0.01$ \\
\hline Mean FA of Meynert's nucleus white matter & $0.65 \pm 0.01$ & $0.60 \pm 0.01$ \\
\hline Grey matter density in Meynert's nucleus & $0.59 \pm 0.01$ & \\
\hline
\end{tabular}

significant also taking into account age and whole-brain skeletonized white matter mean FA $(r=0.52, p<0.001)$ using a partial correlation approach. In other words, lower Ch4 FA values, i.e. less organized white matter tracts in the MN, showed a significant tendency to correlate with lower IQ.

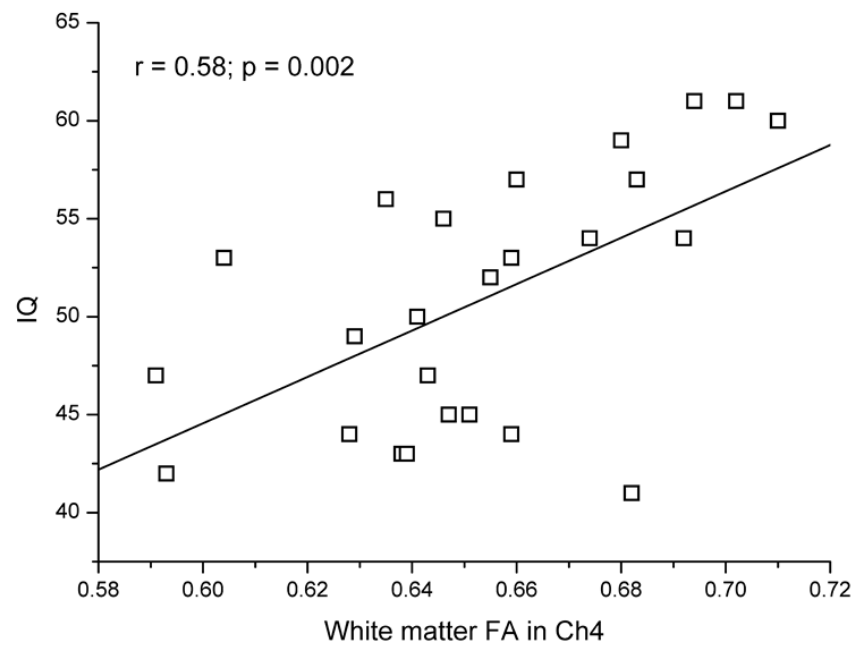

Figure 1: Correlation between mean FA values of the skeletonized white matter included in the Meynert's nucleus and non-verbal IQ in the ASD group.

\section{DISCUSSION}

This study aimed to evaluate if ASD relate to disruptions of the cortical cholinergic projections, which project from the nucleus basalis of Meynert (MN) to the cerebral cortex. We showed a significant structural integrity reduction of those white matter bundles included in the Meynert's nucleus complex (MN) in subjects with low-functioning Autism Spectrum Disorders (ASD), compared to age-matched controls. We reported a significant correlation between Ch4 mask white matter mean FA values and non-verbal IQ scores in subjects with ASD (independently from whole-brain white matter mean FA). We did not find any significant differences in Ch4 grey matter density between ASD subjects and controls.

Our results are in line with a recent study, which found an association between lower FA values in the MN cortical pathway and the "cholinergic deficiency syndrome", a cluster of symptoms that include agitation, anxiety, apathy, delusions, hallucinations, and irritability; symptoms that are often present in people with ASD as well [21].

Previously published post-mortem studies on cholinergic function in autism showed a significant reduction of nicotinic acetylcholine receptor expression in cortical, cerebellar and deep grey areas without alterations in acetylcholinesterase activity, suggesting a post-synaptic deficit of cholinergic transmission $[4,6]$. In vivo studies, on the other hand, showed reduction of acetylcholinesterase activity in the fusiform gyrus (i.e. a cholinergic pre-synaptic deficit), which might correlate with social impairment [7].

Coherently with these studies, our data point to the integrity of Meynert's nucleus grey structures, but also suggest structural alterations of cholinergic projections, as shown by the reduced Ch4 white matter mean FA values and their correlation with IQ. Moreover, our findings are consistent with other DTI studies, which showed a significant relationship between autistic clinical features and white matter structural alterations $[18,22]$.

Coherently with our results, acetylcholine receptor alterations [23] and cholinergic fibre damage [24] have been shown to correlate with cognitive deficits in several neurodegenerative conditions. Alterations in the cholinergic system have been observed also in 
mouse models of Rett syndrome, a condition which shows several contact points with autism [25]. As an upshot of these observations, acetylcholine increase has been used in animal models of autism with the aim of reducing cognitive rigidity and social deficiencies [3].

Interestingly, the MN has been also identified - in the rhesus macaque brain - as one of the main localizations of oxytocin receptors [26]. In fact, several observations point today towards the existence of oxytocin dysregulations in ASD [27].

The main limitation of our study is the possibility of a type I error, based on the relatively low number of patients; thus, further studies including higher number of patients are warranted. Furthermore, we studied here only an adult population of people with ASD. Therefore, the presented result cannot be automatically broadened to younger populations. In addition to these limitations, we have also to recall that all ASD subjects in our study presented with mental retardation as well. For that reason, one possible interpretation of our findings could also be the existence of qualitative white matter differences between ASD people with and without mental retardation. Future studies comparing ASD subjects with and without mental retardation, as well as with non-autistic subjects with mental retardation could help to better understand whether the white matter abnormalities shown in this study are more related to mental retardation, to autism or to both. Many possible caveats have to be considered here, as the intertwinement between ASD and mental retardation is until now only poorly understood.

Therefore, further studies investigating the innervation patterns of the human MN are needed, particularly with the advancement in tractography and other high-resolution imaging techniques [28, 29]. There is the need for the $\mathrm{MN}$ to be revisited in imaging studies, particularly because the potential for neuromodulatory treatment targeting the $\mathrm{MN}$ has to be better appraised and analyzed. These studies might improve also our understanding of the pathological basis of the varying IQs in different people with ASD $[30,31]$.

In conclusion, our data, based on in-vivo assessment of cholinergic nuclei structural properties in subjects with ASD, did not show grey matter alterations of the Meynert's Nucleus in low-functioning autism, but point to white matter abnormalities in the Meynert's Nucleus complex as a possible contributory cause to ASD symptomatology. These findings provide some anatomical support for the hypothesis that disruptions of the cholinergic projections from the $\mathrm{MN}$ might play a role in the development of low-functioning ASD. Larger, longitudinal studies are needed to confirm these observations and to explore the clinical usefulness of MRI-based measures of Ch4 integrity in identifying people who might benefit from a new generation of cholinergic treatments.

\section{ACKNOWLEDGEMENTS}

This work was supported by the "Fondazione G.B. Baroni Onlus".

\section{CONFLICT OF INTERESTS}

M. Pardini received research support from Novartis. All other authors do not report any conflict of interest.

\section{ABBREVIATIONS} $\begin{aligned} \text { ADOS }= & \text { Autism Diagnostic Observation Schedule } \\ & (\text { ADOS })\end{aligned}$

ADI-R = Autism Diagnostic Interview - Revised

$\mathrm{FA}=$ fractional anisotropy

\section{REFERENCES}

[1] American Psychiatric Association. Diagnostic and statistical manual of mental disorders. 5th ed. Arlington, VA: American Psychiatric Association 2013.

[2] Chez MG, Aimonovitch M, Buchanan T, Mrazek S, Tremb RJ. Treating autistic spectrum disorders in children: utility of the cholinesterase inhibitor rivastigmine tartrate. J Child Neurol 2004; 19: 165-9.

[3] Karvat G, Kimchi T. Acetylcholine elevation relieves cognitive rigidity and social deficiency in a mouse model of autism. Neuropsychopharmacology 2014; 39: 831-40. https://doi.org/10.1038/npp.2013.274

[4] Lee M, Martin-Ruiz C, Graham A, Court J, Jaros E, Perry R, et al. Nicotinic receptor abnormalities in the cerebellar cortex in autism. Brain 2002; 125: 1483-95. https://doi.org/10.1093/brain/awf160

[5] Wang L, Almeida LE, Spornick NA, Kenyon N, Kamimura S, Khaibullina $A$, et al. Modulation of social deficits and repetitive behaviors in a mouse model of autism: The role of the nicotinic cholinergic system. Psychopharmacology 2015; 232: 4303-16.

https://doi.org/10.1007/s00213-015-4058-z

[6] Perry EK, Lee ML, Martin-Ruiz CM, Court JA, Volsen SG, Merrit $\mathrm{J}$, et al. Cholinergic activity in autism: abnormalities in the cerebral cortex and basal forebrain. Am J Psychiatry 2001; 158: 1058-66. https://doi.org/10.1176/appi.ajp.158.7.1058

[7] Suzuki K, Sugihara G, Ouchi Y, Nakamura K, Tsujii M, Futatsubashi $\mathrm{M}$, et al. Reduced acetylcholinesterase activity in the fusiform gyrus in adults with autism spectrum disorders. Arch Gen Psychiatry 2011; 68: 306-13. https://doi.org/10.1001/archgenpsychiatry.2011.4

[8] Wang L, Almeida LE, Nettleton M, et al. Altered nocifensive behavior in animal models of autism spectrum disorder: The 
role of the nicotinic cholinergic system. Neuropharmacology 2016; 111: 323-334.

https://doi.org/10.1016/j.neuropharm.2016.09.013

[9] McGaughy J, Everitt BJ, Robbins TW, Sarter M. The role of cortical cholinergic afferent projections in cognition: impact of new selective immunotoxins. Behav Brain Res 2000; 115: 251-63.

https://doi.org/10.1016/S0166-4328(00)00262-X

[10] Luongo FJ, Horn ME, Sohal VS. Putative microcircuit-level substrates for attention are disrupted in mouse models of autism. Biol Psychiatry 2016; 79: 667-75. https://doi.org/10.1016/j.biopsych.2015.04.014

[11] Van Schalkwyk GI, Lewis AS, Qayyum Z, Koslosky K, Picciotto MR, Volkmar FR. Reduction of aggressive episodes after repeated transdermal nicotine administration in a hospitalized adolescent with Autism Spectrum Disorder. J Autism Dev Disord 2015; 45: 3061-6.

[12] Mesulam MM, Mufson EJ, Levey Al, Wainer BH. Cholinergic innervation of cortex by the basal forebrain: cytochemistry and cortical coa, diagonal band nuclei, connections of the septal areleus basalis (substantia innominata), and hypothalamus in the rhesus monkey. J Comp Neurol 1983; 214(2): 170-97.

https://doi.org/10.1002/cne.902140206

[13] Lord C, Risi S, Lambrecht L, Cook EH Jr, Leventhal BL, DiLavore PC, et al. The autism diagnostic observation schedule-generic: a standard measure of social and communication deficits associated with the spectrum of autism. J Autism Dev Disord 2000; 30: 205-23. https://doi.org/10.1023/A:1005592401947

[14] Lord C, Rutter M, Le Couteur A. Autism Diagnostic InterviewRevised: a revised version of a diagnostic interview for caregivers of individuals with possible pervasive developmental disorders. J Autism Dev Disord 1994; 24: 659-85. https://doi.org/10.1007/BF02172145

[15] Leiter RG. Instruction Manual for the Leiter International Performance Scale. Wood Dale, II: Stoelting Co 1979.

[16] Zaborszky L, Hoemke L, Mohlberg H, Schleicher A, Amunts $\mathrm{K}$, Zilles K. Stereotaxic probabilistic maps of the magnocellular cell groups in human basal forebrain. Neurolmage 2008; 42: 1127-41. https://doi.org/10.1016/j.neuroimage.2008.05.055

[17] Smith SM, Jenkinson M, Woolrich MW, Beckmann CF, Behrens TE, Johansen-Berg $\mathrm{H}$, et al. Advances in functional and structural MR image analysis and implementation as FSL. Neurolmage 2004; 23 Suppl 1: S208-19. https://doi.org/10.1016/i.neuroimage.2004.07.051

[18] Pardini M, Garaci FG, Bonzano L, Roccatagliata L, Palmieri MG, Pompili $\mathrm{E}$, et al. White matter reduced streamline coherence in young men with autism and mental retardation. Eur J Neurol 2009; 16: 1185-90. https://doi.org/10.1111/j.1468-1331.2009.02699.x

[19] Smith SM, Jenkinson M, Johansen-Berg $H$, Rueckert $D$, Nichols TE, Mackay CE, et al. Tract-based spatial statistics: voxelwise analysis of multi-subject diffusion data. Neurolmage 2006; 31: 1487-505. https://doi.org/10.1016/j.neuroimage.2006.02.024
[20] Zhang Y, Brady M, Smith S. Segmentation of brain MR images through a hidden Markov random field model and the expectation-maximization algorithm. IEEE Trans Med Imaging 2001; 20: 45-57.

\section{https://doi.org/10.1109/42.906424}

[21] van Dalen JW, Caan MW, van Gool WA, Richard E. Neuropsychiatric symptoms of cholinergic deficiency occur with degradation of the projections from the nucleus basalis of Meynert. Brain Imaging Behav 2016; Oct 27. [Epub ahead of print].

[22] Pardini M, Elia M, Garaci FG, Guida S, Coniglione F, Krueger $\mathrm{F}$, et al. Long-term cognitive and behavioral therapies, combined with augmentative communication, are related to uncinate fasciculus integrity in autism. J Autism Dev Disord 2012; 42: 585-92.

https://doi.org/10.1007/s10803-011-1281-2

[23] Deutsch SI, Burket JA, Urbano MR, Benson AD. The $\alpha_{7}$ nicotinic acetylcholine receptor: A mediator of pathogenesis and therapeutic target in autism spectrum disorders and Down syndrome. Biochem Pharmacol 2015; 97: 363-77. https://doi.org/10.1016/j.bcp.2015.06.005

[24] Bocti C, Swartz RH, Gao FQ, Sahlas DJ, Behl P, Black SE, et al. A new visual rating scale to assess strategic white matter hyperintensities within cholinergic pathways in dementia. Stroke 2005; 36: 2126-31

https://doi.org/10.1161/01.STR.0000183615.07936.b6

[25] Oginsky MF, Cui N, Zhong W, Johnson CM, Jiang C. Alterations in the cholinergic system of brain stem neurons in a mouse model of Rett syndrome. Am J Physiol Cell Physio 2014; 307(6): C508-C520.

[26] Freeman SM, Inoue K, Smith AL, Goodman MM, Young LJ. The neuroanatomical distribution of oxytocin receptor binding and mRNA in the male rhesus macaque (Macaca mulatta). Psychoneuroendocrinology 2014; 45: 128-41.

\section{https://doi.org/10.1016/j.psyneuen.2014.03.023}

[27] Emberti Gialloreti L, Benvenuto A, Benassi F, Curatolo P. Are caesarean sections, induced labor and oxytocin regulation linked to Autism Spectrum Disorders? Med Hypotheses 2014; 82: 713-8.

https://doi.org/10.1016/j.mehy.2014.03.011

[28] Kilimann I, Grothe M, Heinsen $\mathrm{H}$, et al. Subregional basa forebrain atrophy in Alzheimer's disease: a multicentre study. J Alzheimers Dis 2014; 40: 687-700.

[29] Kim HJ, Lee JE, Shin SJ, Sohn YH, Lee PH. Analysis of the substantia innominata volume in patients with Parkinson's disease with dementia, dementia with Lewy bodies, and Alzheimer's disease. J Mov Disord 2011; 4: 68-72. https://doi.org/10.14802/jmd.11014

[30] Gratwicke J, Kahan J, Zrinzo L, et al. The nucleus basalis of Meynert: a new target for deep brain stimulation in dementia? Neurosci Biobehav Rev 2013; 37: 2676-88. https://doi.org/10.1016/j.neubiorev.2013.09.003

[31] Kuhn J, Hardenacke K, Lenartz D, et al. Deep brain stimulation of the nucleus basalis of Meynert in Alzheimer's dementia. Mol Psychiatry 2014; 20(3): 353-60. https://doi.org/10.1038/mp.2014.32

\section{DOI: http://dx.doi.org/10.6000/2292-2598.2016.04.04.1}

(c) 2016 Pardini et al.; Licensee Lifescience Global.

This is an open access article licensed under the terms of the Creative Commons Attribution Non-Commercial License (http://creativecommons.org/licenses/by-nc/3.0/) which permits unrestricted, non-commercial use, distribution and reproduction in any medium, provided the work is properly cited. 